\title{
Duração da Neoplasia Intra-Epitelial e do Carcinoma Invasor do Colo Uterino: Estudo Epidemiologico
}

\author{
Duration of Intraepithelial Neoplasia and Invasive Carcinoma \\ of the Cervix in Relation to Age at Diagnosis
}

Luiz Carlos Zeferino, Aloisio José Bedone

Anibal Faúndes, Nadir Oyakawa

\begin{abstract}
RESUMO
Objetivo: estimar a duração e o tempo de evolução da neoplasia do colo uterino, a partir da infecção por papilomavirus humano (HPV) até as formas invasoras avançadas, tomando como parâmetro a idade média ao diagnóstico.

Método: estudo observacional-transversal que incluiu 1.177 mulheres com infecção por HPV, 1.561 com neoplasia intra-epitelial cervical (NIV) e 773 com carcinoma invasor.

Resultados: não houve diferença estatisticamente significante entre as médias de idade ao diagnóstico da NIC 1 e NIC 2. A duração da NIC 2 foi 2,2 anos e da NIC 3 foi 10,3 anos, sendo 4,1 anos como displasia grave e 6,2 anos como carcinoma in situ (CIS). A duração da lesão intra-epitelial escamosa de alto grau foi 12,5 anos e do carcinoma invasor estádio Ia, Ib e II foram, respectivamente, 3,0, 2,7 e 3,7 anos.

Conclusões: de acordo com os resultados deste estudo, as NIC 1 e NIC 2 originam-se diretamente da infecção por HPV e a maioria das NIC 2 seria uma lesão transiente. A lesão de maior duração é o CIS e o tempo médio do periodo subclinico da neoplasia do colo uterino é de 18,2 anos. Estes resultados são discutidos em função do conhecimento mais atual da história natural do carcinoma do colo uterino e de outros estudos que estimaram a duração desta neoplasia.
\end{abstract}

PALAVRAS-CHAVE: Rastreamento para câncer. Colpocitologia. Colo do útero: lesões préneoplásicas. Colo do útero: carcinoma. Câncer: epidemiologia.

Departamento de Tocoginecologia (DTG) da Faculdade de Ciências Médicas (FCM) e Centro de Atenção Integral à Saúde da Mulher (CAISM) da Universidade Estadual de Campinas (UNICAMP).

Correspondência:

Luiz Carlos Zeferino

UNICAMP - Diretoria Executiva do CAISM

Rua Alexander Fleming, 101 - Cidade Universitária

"Zeferino Vaz"

13.081-970 - Campinas - SP

Fone (019) 788-9402/ Fax: (019) 289-5935

\section{Introdução}

Quando Richart ${ }^{12}$ descreveu a história natural da neoplasia intra-epitelial cervical (NIC), estava convencido de que a displasia era simplesmente um câncer inicial e que, embora algumas displasias leves pudessem regredir 
espontaneamente, a grande maioria evoluiria para carcinoma in situ (CIS) e carcinoma invasor. Hoje sabe-se que as NIC representam um grupo de lesões que têm alta possibilidade de regredir espontaneamente, podem permanecer estáveis por tempo desconhecido e um contigente muito menor pode progredir até tornar-se carcinoma invasor ${ }^{10}$. Esta dinâmica evolutiva das lesões precursoras tem implicações no planejamento do rastreamento e das ações diagnósticas e terapêuticas.

Não é possível determinar com precisão o tempo de evolução das lesões intra-epiteliais até tornarem-se carcinoma invasor, pois aspectos éticos restringem estudos longitudinais com estes objetivos. Assim, alguns estudos estimaram a duração do CIS com base na diferença da idade média da incidência e também a partir de dados de incidência e prevalência ${ }^{1,2,4,5}$. Mais recentemente, alguns pesquisadores elaboraram modelos matemáticos mais complexos, tendo como base o conhecimento mais atual da história natural do carcinoma do colo uterino, as ações de rastreamento realizadas ao longo do tempo e dados de incidência e prevalência ${ }^{6,9}$.

Os estudos mais antigos têm importantes restrições porque partiram de suposições que hoje sabemos não serem verdadeiras, pela complexidade da história natural do carcinoma do colo uterino. Os estudos mais recentes usaram conjuntos de dados que variam muito entre diferentes países, o que inviabiliza a adaptação destes modelos, principalmente para subsidiar o rastreamento do câncer do colo uterino. Destaca-se que quase a totalidade destes estudos focalizaram o CIS e faltam mais informações sobre as demais lesões precursoras e o carcinoma invasor subclínico, que são detectados por meio do rastreamento e têm bom prognóstico. Assim, o objetivo deste estudo foi analisar a duração e evolução da neoplasia do colo uterino, a partir da infecção por HPV até as formas invasoras avançadas, tomando como parâmetro a idade média ao diagnóstico.

\section{Métodos}

Este estudo foi do tipo observacionaltransversal, que incluiu 3.511 mulheres, sendo 1.561 com NIC, 773 com carcinoma invasor e 1.177 com diagnóstico de infecção por HPV, atendidas num hospital universitário de referência regional. Optou-se por selecionar um periodo em que as ações de rastreamento de câncer do colo uterino eram incipientes e, conseqüentemente, pouco interferiram na prevalência das lesões intra- epiteliais e invasoras subclínicas. Assim, os dados foram coletados retrospectivamente de prontuários de mulheres atendidas entre 1972 e 1989. Os exames colpocitológicos eram realizados oportunamente, a partir do início da atividade sexual, em mulheres que procuravam serviços de saúde, incluindo hospital universitário, por qualquer motivo. O diagnóstico de infecção por HPV foi estabelecido com base no exame colpocitológico ou histológico e as demais lesões intra-epiteliais e invasoras com base no diagnóstico histológico.

As idades médias ao diagnóstico foram calculadas para a infecção por HPV, NIC, displasia grave, CIS e carcinomas invasores estádios clínicos Ia, Ib, II e III. O estadiamento do carcinoma invasor adotado naquele período considerou como estádio clínico Ia a invasão do estroma inferior a $5 \mathrm{~mm}$ de profundidade. A duração média de cada lesão foi estimada subtraindo a idade média de seu diagnóstico da idade média da lesão subseqüente. Por exemplo: a duração do carcinoma invasor estádio Ib correspondeu à diferença entre idade média ao diagnóstico dos estádios II e Ib. Portanto, não foi possivel calcular a duração ou tempo de evolução até o carcinoma invasor estádio III, pois não tínhamos informações sobre os casos estádios IV e dos óbitos de mulheres sem qualquer intervenção terapêutica. O método adotado para estimar a duração das fases é tão mais preciso quanto maiores forem as subdivisões da seqüência do processo evolutivo da neoplasia. Assim, a duração média de NIC 3 foi estimada subclassificando-a em displasia grave e CIS. Compondo os diagnósticos, estimou-se a duração da lesão intra-epitelial escamosa de alto grau (LIE-AG), segundo o Sistema de Bethesda. As idades médias foram comparadas duas a duas, entre diagnósticos adjacentes, utilizando-se o teste t de Student. As idades médias do conjunto das lesões foram comparadas empregando-se a análise de variância.

\section{Resultados}

A idade média ao diagnóstico foi maior para as lesões neoplásicas mais graves e a análise de variância mostrou que as diferenças foram estatisticamente significativas. Destacou-se o fato de a idade média da NIC 2 ser menor do que a da NIC 1, ainda que a diferença entre elas não tenha sido estatisticamente significativa. A idade média ao diagnóstico da infecção por HPV foi menor do que as idades médias da NIC 1 e NIC 2. Também não foi encontrada significância na diferença entre 
as idades médias das mulheres com carcinoma invasor E-Ia e carcinoma invasor E-Ib. Todas as demais comparações de duas idades médias de diagnósticos adjacentes foram estatisticamente significativas (Tabela 1).

Tabela 1 - Média de idade no diagnóstico da infecção por HPV, neoplasia intra-epitelial cervical $(\mathrm{NIC})$ e carcinoma invasor do colo uterino $(n=3.511)$

\begin{tabular}{ccccc}
\hline & & & & \\
Diagnóstico $^{1}$ & $\mathrm{n}$ & $\%$ & ${\text { Idade }(\text { média })^{2}}$ & $\mathrm{t}$ de Student \\
\hline HPV & 1.177 & 33,5 & 26,5 & $\mathrm{p}<0,0001$ \\
NIC1 & 216 & 6,2 & 31,2 & N.S. \\
NIC2 & 383 & 10,9 & 30,8 & $\mathrm{p}<0,0001$ \\
NIC3 & 962 & 27,4 & 36,0 & $\mathrm{p}<0,0001$ \\
E-Ia & 77 & 2,2 & 43,3 & $\mathrm{~N} . \mathrm{S}$. \\
E-Ib & 148 & 4,2 & 46,3 & $\mathrm{p}=0,05$ \\
E-II & 188 & 5,4 & 49,0 & $\mathrm{p}=0,001$ \\
E-III & 360 & 10,3 & 52,7 & \\
\hline
\end{tabular}

${ }^{1}$ Os carcinomas invasores foram classificados por estádio clínico.

${ }^{2}$ Análise de variância: comparação do conjunto das idades médias: $p<0,0001$.

${ }^{3}$ Teste t de Student: comparação com a idade média do diagnóstico subseqüênte.

A diferença entre as idades médias da infecção por HPV e o carcinoma invasor E-III, que corresponderia aos dois extremos do processo evolutivo desta neoplasia, foi de 26,2 anos. A duração média da infecção por HPV ou o intervalo médio entre o seu diagnóstico e o da NIC 1 foi de 4,7 anos, ao passo que para a NIC 2 foi de 4,3 anos. Não foi possivel estimar a duração da NIC 1 porque a sua idade média ao diagnóstico foi praticamente superposta à da NIC 2. A NIC 3 foi a lesão com maior duração média, estimada em 10,3 anos - 4,2 anos como displasia grave e 6,1 anos como CIS - que corresponde à transição da fase intra-epitelial para a invasora (Tabela 2).

Tomando como referencial o Sistema de Bethesda, não foi possivel estimar a duração da lesão intra-epitelial escamosa de baixo grau porque não foi possivel estimar a duração da NIC 1. A duração das lesões intra-epiteliais escamosas de alto grau foi de 12,5 anos (Tabela 2).

\section{Discussão}

De acordo com os resultados deste estudo podemos admitir que a NIC 1 e a NIC 2 derivam diretamente da infecção por HPV, pois as idades
Tabela 2- Duração das fases evolutivas da neoplasia do colo uterino, a partir da infecção por HPV até carcinoma invasor estádio clínico II.

Fase/diagnóstico Duração (anos)

\begin{tabular}{lc}
\hline HPV & $4,7^{1} / 4,3^{2}$ \\
NIC 1 & - \\
NIC 2 & 2,2 \\
NIC 3 (displ. grave) & $4,2 \square$ NIC 3: 10,3 anos \\
NIC 3 (CIS) & $6,1]$ LIE $-\mathrm{AG}^{3}:$ \\
E-I a & $3,0,5$ anos \\
E-I b & 2,7 \\
E-II & 3,7
\end{tabular}

${ }^{1}$ Duração da infecção por HPV em relação a NIC 1.

${ }^{2}$ Duração da infecção por HPV em relação a NIC 2.

${ }^{3}$ Lesão intra-epitelial escamosa de alto grau: Sistema de Bethesda.

médias ao diagnóstico destas lesões foram estatisticamente semelhantes. Ainda que a NIC 1 pudesse transformar-se em NIC 2, e vice-versa, esta não seria a via preferencial de evolução destas lesões. Por outro lado, se admitirmos que toda NIC 2 deva passar pela fase de NIC 1, esta passagem seria muito rápida. Os fatores determinantes da expressão destas lesões ainda não são suficientemente conhecidos; todavia, sabe-se que os HPV de alto risco, em especial os tipos 16 e 18, são mais prevalentes na NIC 2 e estão associados ao maior potencial evolutivo das lesões. O Sistema de Bethesda classifica as NIC 1 e NIC 2 como duas lesões distintas, o que é coerente com a suposição de que poderiam originar-se diretamente da infecção por HPV (National Cancer Institute Workshop, 1989) ${ }^{8}$.

Outros estudos relataram idades médias ao diagnóstico semelhantes para as NIC 1 e NIC 2. Sadeghi et al. ${ }^{13}$, num estudo entre mulheres jovens e adolescentes da Califórnia, Estados Unidos, encontraram 25,1 e 25,3 anos de idade média ao diagnóstico, respectivamente, para NIC 1 e NIC 2. Wright e Riopelle ${ }^{16}$, em Ontário, Canadá, obtiveram para NIC 1 e NIC 2, respectivamente, 29,4 e 28,5 anos de idade média ao diagnóstico. Zeferino et al. ${ }^{17}$ em Campinas, Brasil, observaram que as idades médias ao diagnóstico da NIC 1 e NIC 2 foram, respectivamente, 31,4 e 31,3 anos. Disaia e Creasman ${ }^{3}$ mostraram que o tempo de transição do epitélio normal para a displasia leve-moderada em suas pacientes foi de 1,62 anos, não tendo sido identificado separadamente o tempo para cada uma das lesões.

O tempo médio estimado da duração das LIEAG é 12,5 anos. A fase com menor tempo de 
duração é NIC 2, ou seja, quando estas lesões não regridem, evoluem num pequeno intervalo, de em média 2,2 anos, para NIC 3 (displasia grave). Neste sentido, o estudo prospectivo de Kuopio, na Finlândia, mostrou que as taxas de regressão das NIC aumentam com o tempo de seguimento, ao passo que as NIC que progridem clinicamente $o$ fazem muito rapidamente, quase que invariavelmente durante os dois primeiros anos após o diagnóstico, pois as taxas de progressão observadas permaneceram praticamente inalteradas, ao redor de $14 \%$, após 25 meses de seguimento ${ }^{15}$. Também Parkin et al. ${ }^{11}$, por meio de modelos de simulação do programa de rastreamento do câncer do colo uterino, concluiu que as displasias são condições relativamente transientes, pois a maioria regride e a duração mediana é somente de dois a três anos.

Uma vez que a mulher tenha adquirido uma NIC 3, esta lesão pode permanecer nesta fase por um longo período, que neste estudo foi de 10,3 anos, sendo que 4,2 anos corresponderam à displasia grave e 6,1 anos ao CIS. De acordo com os resultados deste estudo, a displasia grave e o CIS podem ser considerados como fases cronologicamente distintas, ainda que, do ponto de vista prático, a abordagem assistencial usualmente é a mesma e as classificações de Richart ${ }^{12}$ e de Bethesda ${ }^{8}$ consideram-nas como uma mesma lesão.

A maioria dos estudos sobre a duração da neoplasia do colo uterino analisou o CIS e as estimativas variaram largamente. Souen e Salvatore ${ }^{14}$, com base na idade média ao diagnóstico, estimaram a duração do CIS em 6,3 anos e identificaram seis estudos, publicados entre 1961 e 1976, cujas estimativas da duração do CIS variaram de 4,2 a 10,5 anos. Zeferino et al. ${ }^{17}$ relataram médias de idade de 37,5 e 45,5 anos, quando do diagnóstico, respectivamente, para NIC 3 e carcinoma invasor E-I; o que indica uma duração média da fase NIC 3 de oito anos. Outros estudos que utilizaram métodos baseados na análise da prevalência e da incidência do CIS, obtiveram valores que variaram entre oito e 14 anos ${ }^{1,2}$. Gustafsson e Adami ${ }^{6}$ elaboraram um modelo que considera os resultados do rastreamento do câncer do colo uterino e estimaram a duração do CIS em 13,3 anos; porém, em mulheres que não realizaram controles colpocitológicos periódicos este tempo foi de quatro anos. As variações observadas entre as estimativas podem ser decorrentes das diferenças entre os métodos adotados, qualidade das informações e também de diferenças epidemiológicas reais entre as populações de estudo.

A duração do carcinoma invasor diminuiu do estádio Ia para o estádio II, o que pode ser explicado pelo crescimento quase exponencial do volume do tumor invasivo. A média de idade quando do diagnóstico do carcinoma invasor estádio Ib foi maior do que Ia, ainda que a diferença não tivesse sido estatisticamente significante, o que se deveu, provavelmente, ao pequeno número de casos nestes estádios. O intervalo entre a infecção por HPV e o carcinoma invasor estádio III foi de 26,2 anos, resultado muito semelhante ao encontrado por Meisels e Morin ${ }^{7}$, que foi de 27,45 anos entre o condiloma sem atipias e o carcinoma invasor.

Para que os cálculos de idade média fossem mais precisos, seria necessário que a cobertura do rastramento citológico fosse igual em todas as faixas etárias. Como a cobertura do rastreamento foi cerca de $30 \%$ maior nas mulheres entre 20 e 29 anos do que nas faixas etárias entre 30 e 59 anos, as médias de idade das pacientes com infecção por HPV, NIC 1, NIC 2 e displasia grave tenderam a ser um pouco menor que as reais, pois são as lesões mais prevalentes neste grupo etário. As idades médias calculadas para os estádios do carcinoma invasor são próximas das reais, pois nas faixas etárias de maior prevalência destas lesões a cobertura populacional foi semelhante e os estádios II e III usualmente são clinicamente sintomáticos e não são diagnosticados por meio do rastreamento citológico.

Para que as estimativas da duração das fases evolutivas sejam precisas, o ideal é que as médias de duração de cada uma das fases sejam semelhantes. Assim, como a duração média do CIS foi duas vezes a média de duração do carcinoma invasor E-Ia, este método tendeu a subestimar a duração do CIS. Todavia, este método é preciso quando se considera um período compreendendo várias fases contíguas, pois o que se subtrai de uma fase é acrescido nas outras. Nestas condições, os métodos baseados na prevalência e incidência são mais restritivos, pois é muito difícil elaborar modelos matemáticos que permitam analisar várias fases evolutivas de uma neoplasia. É importante destacar que a regressão espontânea das lesões intra-epiteliais não é uma restrição para o método utilizado, pois para calcular a duração de uma fase, subtrai-se a sua idade média do diagnóstico da idade média da fase seguinte, ou seja, das lesões que evoluíram para a fase seguinte.

Assim, o tempo médio estimado para que uma lesão intra-epitelial fosse detectada antes de se tornar invasora é de 12,5 anos, o que corresponde à duração da LIE-AG, segundo o Sistema de Bethesda. Se incluirmos o carcinoma invasor E-Ia, durante o qual poderia ser detectada uma lesão com percentuais de cura próximos a 100\%, o intervalo seria estendido para 15,5 anos. Se 
incluirmos ainda $\mathrm{E}-\mathrm{Ib}$, o tempo médio total atingiria 18,2 anos em que uma lesão, de bom prognóstico, poderia ser detectada, o que se aproximaria da duração do período subclínico o carcinoma do colo uterino. A maioria das mulheres com carcinoma invasor E-I é assintomática ou não tem sintomas importantes e, conseqüentemente, a maioria destas lesões é detectada por meio dos programas de rastreamento e prevenção de câncer do colo uterino. Estes resultados reforçam o conhecimento de que controles a cada três, cinco ou até mesmo dez anos podem reduzir a mortalidade por carcinoma de colo uterino, em razão do longo tempo de evolução desta neoplasia.

\section{SUMMARY}

Purpose: to estimate the duration of cervical neoplasia from human pappilomavirus (HPV) infection to advanced invasive carcinoma, using as paremeter the mean age of the women at diagnosis.

Methods: this cross-sectional study included 1,177 women with HPV infection, 1,561 with cervical intraepithelial neoplasia (CIN) and 773 with invasive carcinoma.

Results: the mean ages of CIN 1 and CIN 2 on diagnosis were not statistically different. The mean duration of CIN 2 was 2.2 years. The mean duration of CIN 3 was 10.3 years, with 4.1 years as severe dysplasia and 6.2 years as carcinoma in situ (CIS). The mean duration of high grade squamous intraepithelial lesions was 12.5 years. The duration means of invasive carcinoma stages Ia, Ib and II were 3.0, 2.7 and 3.7 years, respectively.

Conclusions: according to the results, CIN 1 and CIN 2 may arise directly from HPV infection and most of these lesions are transient. CIS presented the longest duration and the mean asymptomatic period of cervical neoplasia is 18.2 years. These results were discussed considering the present knowledge of the natural history of cervical carcinoma and other studies on duration of this neoplasia.

KEY WORDS: Cancer: screening. Colpocitology. Uterine cervix: intraepithelial lesion. CIN. Cancer: epidemiology.

\section{Referências}

1. Barron BA, Cahil MC, Richart R. A statistical model of the natural history of cervical neoplastic disease: The duration of carcinoma in situ. Gynecol Oncol 1978; 6:196-205.
2. Coppleson LW, Brown B. Observations on a model of the biology of carcinoma of the cervix: A poor fit between observation and theory. Am J Obstet Gynecol 1975; 112:127-36.

3. Disaia PJ, Creasman WT. Preinvasive disease of the cervix. In: Clinical Gynecologic Oncology. 5th ed. St. Louis-Missouri: Mosby-Year Book, 1997.

4. Dunn JE, Martin PL. Morphogenesis of cervical cancer. Finding from San Diego County Cytology Registry. Cancer 1967; 20: 1899-906.

5. Fidler HK, Boyes DA, Worth AJ. Cervical cancer detection in British Columbia. J Obstet Gynecol 1967; 122: 127-36.

6. Gustafsson L, Adami HO. Natural history of cervical neoplasia: consistent results obtained by an identification technique. Br J Cancer 1989; 60:132-41.

7. Meisels A, Morin C. Human papillomavirus and cancer of the uterine cervix. Gynecol Oncol 1981; 12:111-23.

8. National Cancer Institute Workshop. The Bethesda System for reporting cervical: vaginal cytological diagnoses. JAMA 1989; 262:9311-4.

9. Oortmarssen GJ, Habbema JDF. Epidemiological evidence for age-dependent regression of preinvasive cervical. Br J Cancer 1991; 64:559-65.

10.Östör AG. Natural history of cervical intraepithelial neoplasia: A critical review. Int J Gynecol Pathol 1993; 12:186-92.

11.Parkin DM. A computer simulation model for practical planning of cervical cancer screening programmes. Br J Cancer 1985; 51:551-68.

12. Richart R. Natural history of cervical intraepithelial neoplasia. Clin Obstet Gynecol 1968;10:748-85.

13 - Sadeghi SB, Hsieh EW, Gunn SW. Prevalence of cervical intraepithelial neoplasia in sexually active teenagers and young adults: Results of data analysis of mass Papanicolaou screening of 796,337 women in the United States in 1981. Am J Obstet Gynecol 1984; 148:726-9.

14. Souen JS, Salvatore CA. O colo do útero. In: Précâncer em ginecologia. São Paulo: Editora Manole, 1981. p. 71-150.

15.Syrjänen J. Biological behaviour of cervical intraepithelial neoplasia. In: Franco E, Montosonego J. New developments in cervical cancer screening and prevention. London: Blackwell Science, 1997. p. 93-108.

16.Wright VC, Riopelle MA. Age at beginning of coitus versus chronologic age as a basis for Papanicolaou smear screening: An analysis of 747 cases of preinvasive disease. Am J Obstet Gynecol 1984; 149:824-30.

17.Zeferino LC, Nisida AC, Pinotti JA. Epidemiologia da neoplasia intra-epitelial cervical. Rev Bras Ginecol Obstet 1988; 1:22-33. 